\title{
UTILIDAD DE LAS INVESTIGACIONES MONOGRÁFICAS EN LA URACCAN, NUEVA GUINEA 2003-2009
}

\author{
Oved Zeledón Membreño ${ }^{[1]}$ \\ Francisco Antonio Miranda Villachica ${ }^{[2]}$
}

\section{Resumen}

La investigación permitió conocer el estado actual y utilidad de las monografías existentes en la biblioteca "Luz en la Selva" de la URACCAN, recinto Nueva Guinea, 2003 - 2009. Fue un estudio mixto, con predominancia del enfoque cualitativo, se utilizaron técnicas de recolección de datos como revisión bibliográfica, encuestas y entrevistas, a través de las cuales se obtuvo resultados acerca de la clasificación de las 115 monografías realizadas entre los años 2003-2009, se destaca con mayor aporte las áreas de Ciencias de la Educación, Humanidades y Recursos Naturales y Medio Ambiente, porque exige entre sus requisitos de culminación los trabajos monográficos. El enfoque fue mixto con predominancia cualitativa. Las principales líneas de investigación fueron las de desarrollo socioeconómico ambiental y metodologías de enseñanza-aprendizaje.

La comunidad universitaria tiene conocimiento de la existencia de las monografías en la biblioteca; sin embargo, las formas de divulgación no han sido efectivas. Las monografías han presentado diferentes utilidades: generando proyectos, fuente de otras investigaciones y resolución de problemáticas comunitarias.

Palabras claves: Monografías, investigación, utilidad, clasificación.

\section{Summary}

The investigation allowed knowing the current status and use of the monographs in the library "Luz en la Selva" from URACCAN-Nueva Guinea Campus, during the year 2003 to 2009. The focus of the study was predominantly mixed-qualitative; we used data collection techniques such as literature review, surveys and interviews through which we obtained results on the classification of the 115 monographs conducted between the years 2003-2009. The highest contributions were made by the department of

\footnotetext{
[1] Máster en Docencia Universitaria, Coordinador Ciencias Administrativas e Informática URACCAN, Nueva Guinea. E-mail: obedmembreo@yahoo.com

${ }^{[2]}$ Máster en Docencia Universitaria, Docente URACCAN, Nueva Guinea. E-mail: franciscomiranda70@yahoo.com
} 
Educational Sciences, Humanities and Natural Resources and Environment, because among their requirements of culmination they compel the fulfillment of monographs.

The main lines of research were based on the environmental and socio-economic development and the teaching-learning methodologies.

The members of the university is aware of the existence of the monographs in the library, however the promotion forms have not been effective. The monographs presented different uses: generating projects, source of other researches and how to solve community problems.

Keywords: Monographs, research, use, classification.

\section{Introducción}

La docencia, investigación y la extensión social comunitaria constituyen las funciones fundamentales de la URACCAN. De acuerdo a la visión de Educación Superior, las universidades deberán convertirse en centros de nuevos liderazgos y deberá asentarse sobre bases sólidas en sus funciones básicas de docencia, investigación y proyección social.

La visión anterior se corrobora con el establecimiento de la Dirección de Investigación y Postgrado, instancia responsable de la planificación, organización, monitoreo y dirección de la actividad investigativa, a su vez se vincula con la misión, visión y el modelo pedagógico; por ello, en los currículos de las diferentes carreras ofertadas en el Recinto Universitario Nueva Guinea, presenta, entre otras, la investigación monográfica como una forma de culminación de estudios.

La universidad ha definido su política institucional de investigación en la misión, visión, principios, características, estructura organizativa y áreas investigativas, así mismo en los programas de metodologías de investigación y seminario monográfico, se orientan las investigaciones vinculadas a la política institucional.

La investigación, consecuencia de la práctica universitaria tiene en común denominador: el hacer posible la vinculación integral del estudiante en su formación con la apropiación de la actitud investigativa.

\section{Revisión de literatura}

Fomentar y desarrollar la investigación se define en la Ley No. 89. Ley de Autonomía de las Instituciones de Educación Superior. Aunque Nicaragua posee una cantidad significativa de instituciones de Educación Superior, esto no significa que su capacidad 
de investigación sea alta, ni que el crecimiento de la oferta educativa represente un aumento en la oferta investigativa. La mayoría de las universidades autorizadas después de 1990 centran sus esfuerzos en la docencia.

El número de instituciones de educación terciaria se ha incrementado 5 veces en un período de 15 años, la capacidad de investigación no ha sido proporcional a este crecimiento.

\section{El papel de la investigación universitaria}

Henao (2005, p.1), plantea: "Desde la perspectiva clásica se considera que una de las funciones características de la Universidad es la investigación, la cual hace parte del tríptico que conforma, con la docencia y la extensión, la estructura que soporta los fines consagrados por la tradición a su institucionalidad".

La Conferencia Mundial de Educación Superior (París, 1998), en su artículo No. 1, reafirma esta misión como la mejor manera de contribuir al desarrollo del país, al "promover, generar y difundir conocimientos por medio de la investigación".

\section{Utilidad de la investigación}

La investigación puede cumplir dos propósitos: proporcionar teoría y conocimiento sobre la realidad, el segundo es resolver problemas cotidianos de la sociedad.

Garza (2009) plantea:

Una de las características de la investigación científica y tecnológica es la utilidad, y se demuestra a través de la historia de los grandes avances de la humanidad, desde los tiempos en que el hombre vivió en las cavernas hasta la actualidad en la que la computación es de gran importancia (p.1).

La investigación es un instrumento para poder resolver problemas de la sociedad. La ciencia y tecnología tienen como causas las necesidades del ser humano.

\section{Materiales y métodos}

La investigación se realizó en URACCAN, Recinto Nueva Guinea, RAAS, Nicaragua; bajo un enfoque mixto (cuantitativo y cualitativo), sin embargo predominó el paradigma cualitativo. De acuerdo a la predominancia del estudio, es una investigación descriptiva, estableciendo el comportamiento y caracterizando el fenómeno, a través del estudio de variables. La dimensión del estudio es de corte transversal, correspondiente entre el 2003 al 2009. Las Técnicas e Instrumentos utilizados fueron: revisión documental a monografías existentes en biblioteca, documentación del área de investigación y 
postgrado y revistas universitarias; entrevista a coordinadores, responsable de biblioteca, secretaria académica, encuestas a estudiantes y responsables de entidades afines a los temas.

\section{Unidad de Análisis}

- 115 investigaciones monográficas existentes en biblioteca del recinto URACCAN, Nueva Guinea.

- Estudiantes de todas las modalidades: 310.

- Docentes: 29.

- Coordinadores de áreas del recinto: 3.

- Secretaria académica: 1.

- Responsable de biblioteca: 1.

- Coordinador de la dirección de Investigación y postgrado: 1.

- Dirigentes de entidades de instituciones, ubicados en el área urbana del municipio de Nueva Guinea: 14.

\section{Resultados y discusión}

En el recinto URACCAN Nueva Guinea se registran los trabajos monográfico que ingresan al área de biblioteca, de acuerdo a lo expresado por la responsable de biblioteca del recinto en entrevista realizada el día 27 de junio del 2010, cuando expresó que:

Es requisito para trámites y entrega de títulos a los graduados, llevar constancia de que se ha suministrado en biblioteca la investigación con las revisiones finales, impresa, empastada y en un disco compacto en forma digital.

Cuadro No. 1. Enfoque de monografías en biblioteca, URACCAN Nueva Guinea

\begin{tabular}{|c|c|c|c|c|c|}
\hline Áreas Académicas & Carreras & : & 冚 & 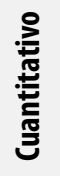 & 을 \\
\hline \multirow{6}{*}{$\begin{array}{c}\text { Ciencias de la Educación y } \\
\text { Humanidades }\end{array}$} & $\begin{array}{l}\text { Ciencias de la Educación con mención en } \\
\text { Español }\end{array}$ & 15 & 03 & 01 & 19 \\
\hline & $\begin{array}{l}\text { Ciencias de la Educación con mención en } \\
\text { Matemática }\end{array}$ & 01 & & 06 & 07 \\
\hline & $\begin{array}{l}\text { Ciencias de la Educación con mención en } \\
\text { Historia }\end{array}$ & 12 & 03 & 02 & 17 \\
\hline & $\begin{array}{l}\text { Ciencias Sociales con mención en Desarrollo } \\
\text { Local }\end{array}$ & 15 & 02 & 03 & 20 \\
\hline & $\begin{array}{l}\text { Ciencias de la Educación con mención en } \\
\text { Biología }\end{array}$ & 05 & 02 & 01 & 8 \\
\hline & Sub-Total & 48 & 10 & 13 & 71 \\
\hline
\end{tabular}




\begin{tabular}{|c|c|c|c|c|c|}
\hline Áreas Académicas & Carreras & 올 & 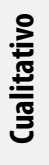 & 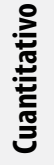 & 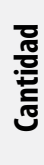 \\
\hline \multirow{3}{*}{ Ciencias Administrativas e Informática } & Administración de Empresas & 01 & & $\mathrm{O} 2$ & 3 \\
\hline & Informática Administrativa & & 01 & & 1 \\
\hline & Sub-Total & 01 & 01 & 02 & 4 \\
\hline \multirow{3}{*}{ Recursos Naturales y Medio Ambiente } & Agroforestal & & & 18 & 18 \\
\hline & Zootecnia & 01 & & 12 & 13 \\
\hline & Sub-Total & 01 & & 30 & 31 \\
\hline Investigación y Postgrado & & 02 & 03 & 04 & 9 \\
\hline \multicolumn{2}{|l|}{ TOTALES } & 52 & 14 & 49 & 115 \\
\hline
\end{tabular}

Fuente: Elaboración propia a partir de la revisión documental en biblioteca "Luz en la Selva".

Las áreas académicas de Ciencias de la Educación, Humanidades y Recursos Naturales y Medio Ambiente, son las que mayor aporte han realizado en monografías, esto se debe, tal como expresan los coordinadores de estas áreas, que la única forma de culminación en las carreas ofertadas es a través de las investigaciones monográficas.

Aunque en la biblioteca existen 115 investigaciones, la cantidad elaborada es mayor ya que hay estudiantes que realizaron todo el proceso de defensa monográfica, pero por diversas razones no han entregado el volumen impreso en la biblioteca del recinto, como en el caso de maestrantes de este recinto que han sido entregadas en otros recintos universitarios de la URACCAN.

Cuadro No. 2. Tipo de monografías existentes en biblioteca URACCAN, Nueva Guinea

\begin{tabular}{|c|c|c|c|c|c|c|c|c|c|c|}
\hline Áreas académicas & 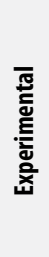 & 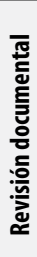 & 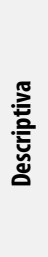 & 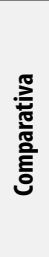 & 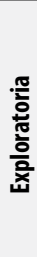 & 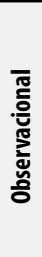 & 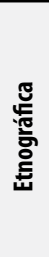 & 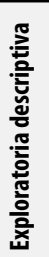 & 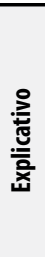 & 흉 \\
\hline $\begin{array}{l}\text { Ciencias de la Educación y } \\
\text { Humanidades }\end{array}$ & 1 & 1 & 54 & & 8 & & 2 & 1 & 4 & 71 \\
\hline $\begin{array}{l}\text { Ciencias Administrativas e } \\
\text { Informática }\end{array}$ & & & 4 & & & & & & & 4 \\
\hline $\begin{array}{l}\text { Recursos Naturales y Medio } \\
\text { Ambiente }\end{array}$ & 22 & 2 & 3 & 1 & 1 & 2 & & & & 31 \\
\hline Investigación y Postgrado & & & 7 & & 2 & & & & & 9 \\
\hline TOTAL & 23 & 3 & 68 & 1 & 11 & 2 & 2 & 1 & 4 & 115 \\
\hline
\end{tabular}

Fuente: Elaboración propia a partir de la revisión documental en biblioteca "Luz en la Selva". 
Cuadro No. 3. Áreas temáticas desarrolladas en las monografías existentes en biblioteca "Luz en la Selva"

\begin{tabular}{|c|l|c|c|l|c|}
\hline No & \multicolumn{1}{|c|}{ Área Temática } & Cantidad & No & \multicolumn{1}{c|}{ Área Temática } & Cantidad \\
\hline 1 & Recurso Agua & 3 & 8 & Autonomía Regional & 2 \\
\hline 2 & Recurso Bosque & 1 & 9 & Salud Comunitaria & 2 \\
\hline 3 & Recurso Suelo & 0 & 10 & Historia & 8 \\
\hline 4 & Biodiversidad & 0 & 11 & Desarrollo Local & 33 \\
\hline 5 & Calidad Ambiental & 5 & 12 & $\begin{array}{l}\text { Metodologías de enseñanza- } \\
\text { aprendizaje }\end{array}$ & 9 \\
\hline 6 & $\begin{array}{l}\text { Desarrollo Socioeconómico } \\
\text { Ambiental }\end{array}$ & 23 & 13 & $\begin{array}{l}\text { Desarrollo Socioeconómico } \\
\text { Agropecuario }\end{array}$ & 19 \\
\hline 7 & Género e interculturalidad & 9 & 14 & $\begin{array}{l}\text { Tecnologías de la Información y } \\
\text { Comunicación }\end{array}$ & 19 \\
\hline
\end{tabular}

Fuente: Elaboración propia.

Tanto el estudiantado como el profesorado expresan en su mayoría que de una u otra forma reciben orientación sobre las líneas de investigación de los temas a indagar puesto que para la aprobación de estas temáticas deben de cumplir algunos requisitos relacionados con la filosofía institucional o el perfil académico de los investigadores.

El coordinador de investigación y postgrado en entrevista realizada el 27 de junio del año 2010 expone que: "Lo que se busca o lo que se garantiza es que estas investigaciones respondan al contexto y los estudiantes puedan realizar sus temas de acuerdo a la pertinencia que establece la universidad en su misión y visión."

\section{Disponibilidad de las investigaciones monográficas}

El 85\% de estudiantes, $100 \%$ de docentes y el 79\% de los encuestados de las instituciones, expresan que se tiene conocimiento de la existencia de investigaciones monográficas en la biblioteca "Luz en la Selva", lo que concuerda con autoridades universitarias y responsable de biblioteca, cuando expresan que la biblioteca es un referente para consultas de información no sólo a nivel interno de la universidad, sino a nivel municipal siendo esta la biblioteca más grande de Nueva Guinea, con mayores recursos bibliográficos, infraestructura, tecnología y accesibilidad.

El profesorado expresa que las monografías están accesibles; sin embargo, el estudiantado e instituciones expresan que hay una limitada accesibilidad, esto muchas veces se debe a las políticas internas del funcionamiento de la biblioteca, puesto que una de ellas es que no se pueden prestar fuera del área de estudio de la biblioteca los recursos bibliográficos que no se cuentan con más de un volumen, y existe un único ejemplar por monografía, en muchas ocasiones los investigadores requieren tener a 
mano estas investigaciones y no tienen tiempo para invertirlo en la biblioteca leyendo una monografía.

\section{Divulgación de las investigaciones monográficas}

Los medios de divulgación de los trabajos monográficos son diversos, variados, sencillos y por lo tanto fáciles de comprender; pero el aprovechamiento de este recurso por parte de la población estudiantil, profesorado y otras instituciones es muy poco, este comportamiento en ocasiones no es por falta de divulgación, sino que los medios por lo que se realiza no están siendo efectivos. Es importante señalar que los docentes juegan un rol primordial en la difusión de estas investigaciones, ya que el 59\% de los estudiantes conocen de estas a través del maestro.

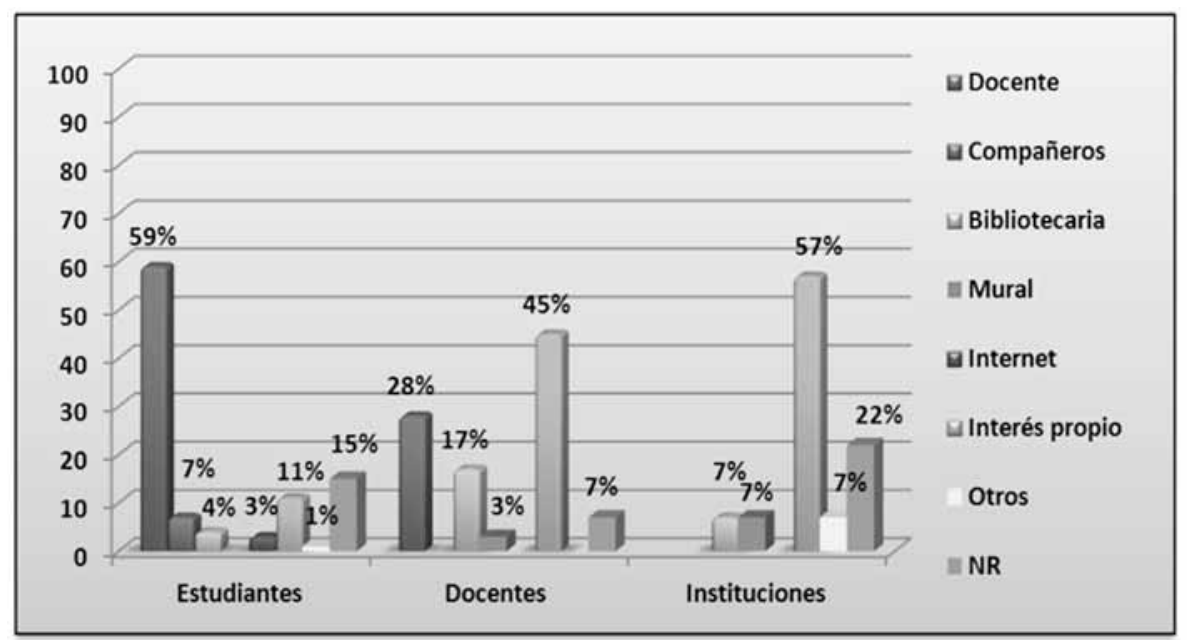

Figura No. 1. Medio por el cual se dio cuenta de la existencia de las monografías en la biblioteca "Luz en la selva", URACCAN, Nueva Guinea.

En relación a la forma en que se ha incentivado el uso de las investigaciones monográficas, tanto el estudiantado como el profesorado expresaron que frecuente y rara vez se les incentiva el uso de las monografías; antes bien. quienes incentivan al estudiantado a utilizar las monografías es el profesorado, la mayoría de las veces se hace uso de ellas cuando se cursan asignaturas relacionadas con metodología de investigación o seminario monográfico, y es ahí donde el maestro orienta su uso, el profesorado expresa que quien los motiva es otro colega o el coordinador, lo que concuerda con lo expresado por las autoridades cuando expresan que la forma de orientar su uso es a través de reuniones o capacitaciones que realizan las autoridades académicas del recinto. 


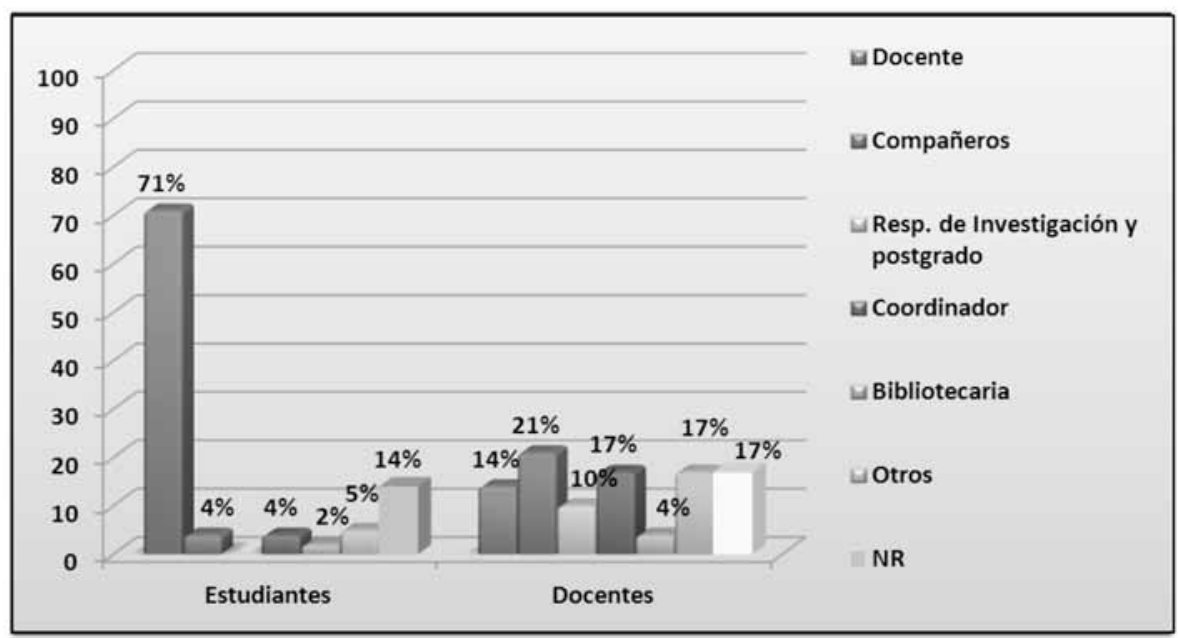

Figura No. 2. Actores que han incentivado la utilización de los trabajos monográficos.

Por otra parte los encuestados de las diferentes instituciones únicamente un $36 \%$ expresaron que han sido invitados a presenciar exposiciones de trabajos de investigación monográfica. Un $79 \%$ de las instituciones encuestadas manifestaron que en la entidad se han realizado investigaciones por el estudiantado de la universidad URACCAN, recinto Nueva Guinea; no obstante, de estos trabajos investigativos únicamente un 50\% opinó que se dieron a conocer los resultados de las mismas. Estos en un 100\% tienen interés en conocer las investigaciones monográficas y estarían dispuestos a participar en las exposiciones de las monografías si se les invita.

\section{Publicaciones de las investigaciones monográficas}

Producto de la revisión documental de los trabajos monográficos, se encontró que de los 115 trabajos realizados, 7 (siete) de estos han sido publicados, lo que equivale a que un $6 \%$, de estos dos han sido publicados a nivel internacional, correspondiendo a $1.73 \%$.

\section{Monografías presentadas en las Jornadas de Desarrollo Científico}

En la Jornada Universitaria de Desarrollo Científico (JUDC), desde el 2004 al 2009 se han presentado 22 trabajos monográficos representando el $18 \%$ de 123 trabajos que deberían estar registrados en biblioteca.

Las JUDC, han jugado un papel importante en los procesos de divulgación, sin embargo existen dificultades en el seguimiento para que estos trabajos puedan ingresar a biblioteca y aportar con sus datos a mejorar las consultas. 


\section{Utilidad de las investigaciones monográficas a nivel interno y externo de la URACCAN}

Los encuestados refieren que consultan de manera esporádica las monografías, en el caso del profesorado y estudiantado coinciden con las autoridades ya que en su mayoría buscan estos tipos de investigaciones únicamente cuando están cursando las asignaturas de metodologías de la investigación, seminario monográfico o elaborando otra investigación.

En el caso de las instituciones es más comprensible, puesto que únicamente hacen consulta de estas informaciones cuando tienen la necesidad de obtener algún dato o valorar algún resultado de cierto tipo de investigación en específico.

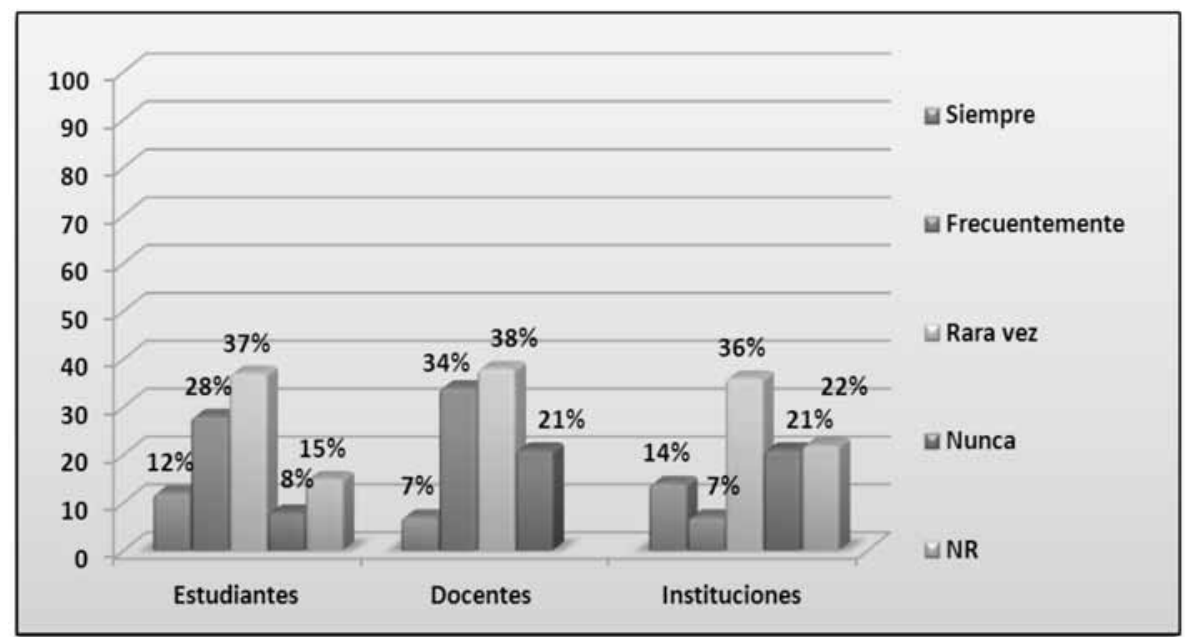

Figura No. 3. Consulta de investigación monográfica en biblioteca URACCAN.

Un $75 \%$ del profesorado contestaron que frecuente y rara vez visitan la biblioteca para consultar la información de las monografías. El 73\% del profesorado orientan al estudiantado frecuentemente y rara vez hacen uso de las investigaciones monográficas, un 79\% respondió que las tareas, trabajos o investigaciones que ellos asignan al estudiantado motivan el uso de libros, únicamente un 7\% motiva el uso de monografías, únicamente un $10 \%$ utilizan las monografías para preparar sus clases, por lo que no se orienta su uso. 


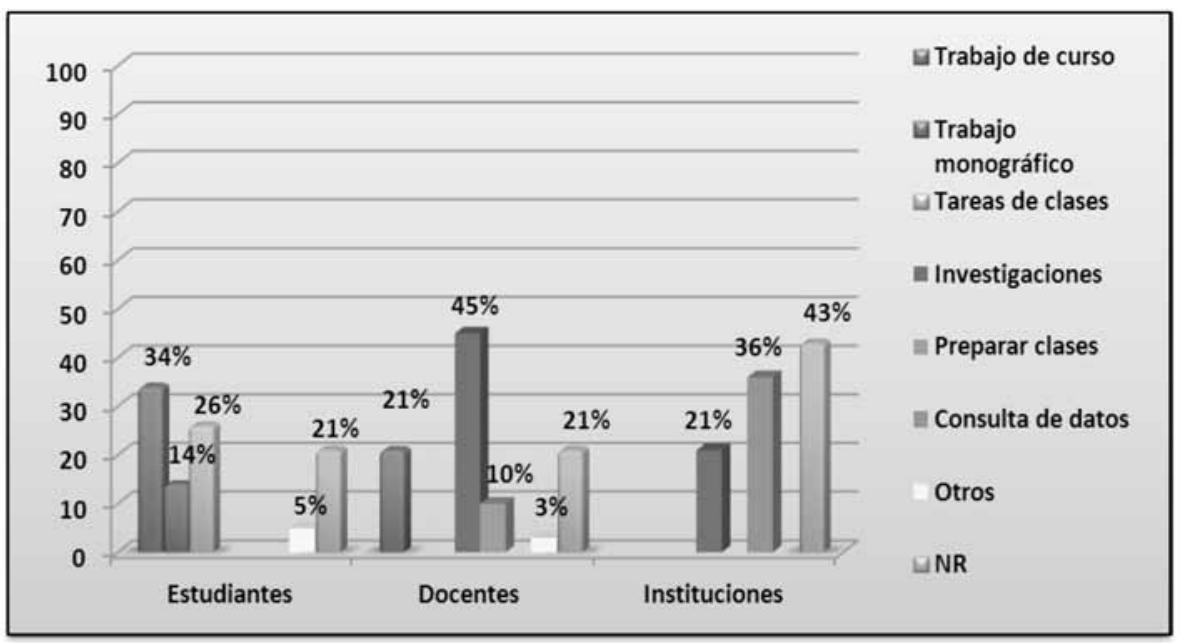

Figura No. 4. Porcentaje de consultas monográficas en biblioteca "Luz en la selva".

Las monografías realizadas no son muy bien aprovechadas, la mayoría de las veces en el caso del estudiantado y profesorado sólo se utilizan para algún tipo de consulta o para citarlas como referencia en algún tipo de investigación que estos estén realizando.

Para las autoridades del recinto estas investigaciones si tienen un uso más significativo, el coordinador de investigación y postgrado manifestó que:

Cuando presentamos un proyecto nos basamos en las investigaciones que hay acá, yo creo que si tienen un interés primordial para la universidad porque aportan información que bien aprovechada creo que podríamos sacarle mucho provecho (Entrevista realizada el 27 de junio del año 2010).

La calidad del contenido de las investigaciones monográficas está avalado por la comisión académica, el aval que realiza el tutor, así como por el jurado calificador que emite un resultado en base a la calidad del trabajo, a la vez el profesorado expresa que el contenido en las investigaciones satisface sus expectativas y los investigadores de instituciones valoran el contenido de estos trabajos como poco significativos y aceptables. Según encuesta, el 63\% del estudiantado respondió que la información de las monografías consultadas ha sido de utilidad en su proceso de formación académica.

La URACCAN no cuenta con un mecanismo, reglamento o ciertos parámetros para mediar la utilidad o calidad de las investigaciones que se desarrollan en el recinto, al respecto el coordinador de Investigación y Postgrado asevera: 
En la política de investigación no queda claro, no están los indicadores para clasificar estas investigaciones bajo parámetros de calidad, igualmente otros parámetros como pertinencia que nuestra política de investigación adolece, es muy limitada en cuanto a establecimiento de indicadores (Entrevista realizada el 27 de junio del año 2010).

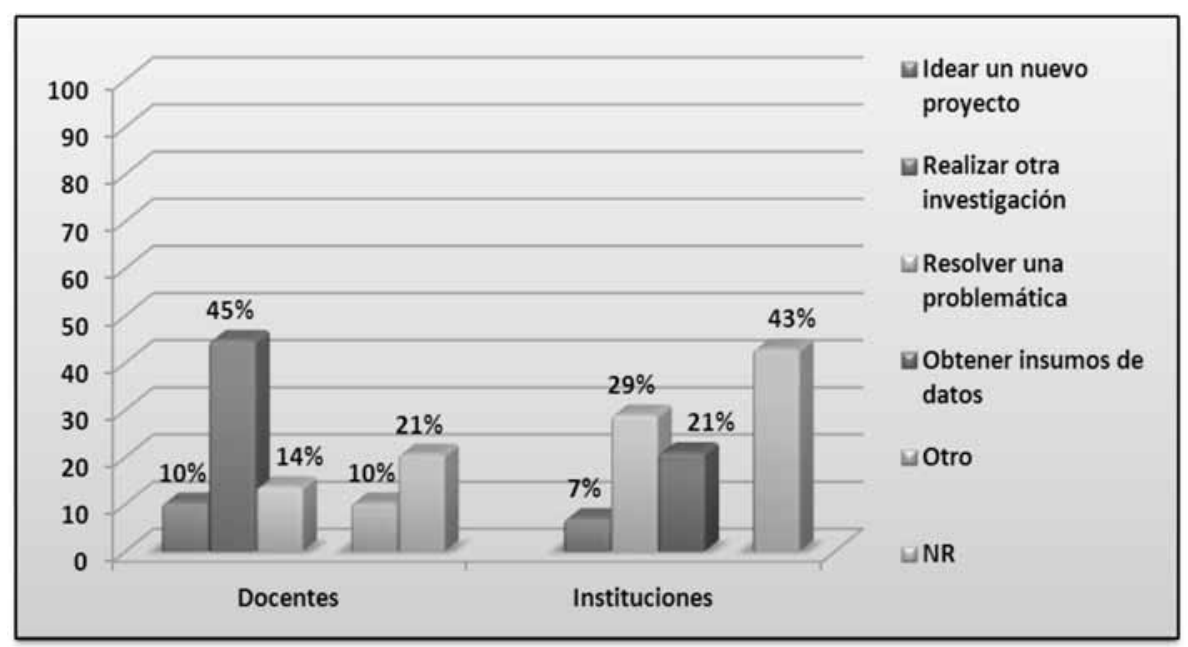

Figura No. 5. Utilización de la información de las monografías consultadas.

Investigaciones de utilidad que han surgido desde el área de ciencias administrativas e informática, son software que ha creado el estudiantado como opción de investigación monográfica, generando diferentes proyectos informáticos como la creación de un sistema de registro académico, este se utiliza para almacenar toda la información concerniente al estudiantado y sus calificaciones y ha sido implementado en todos los recintos de la universidad URACCAN. También se han generado software que se han implementado en diferentes instituciones estatales y entidades no gubernamentales como la Policía Nacional, Fiscalía, APROSAPANG, entre otras.

En el área de Ciencias de la Educación y Humanidades se han generado propuestas didácticas, las cuales han servido para mejorar la enseñanza en Educación Primaria y Secundaria. Desde las carreras de esta área se ha contribuido al rescate de la historia del municipio y hay una serie de investigaciones referidas a la historia de la mayoría de las colonias de Nueva Guinea.

Cabe señalar que el libro, aún no publicado, titulado Nueva Guinea de la oscuridad en la pluvioselva a ciudad de la luz 1965-2008, se realizó basado en varias investigaciones en el campo de historia de las comunidades del municipio y otras instituciones lo han utilizado extrayendo datos para realizar proyectos.

En el área de recursos naturales y medio ambiente, las investigaciones han contribuido de manera significativa al mejoramiento del sistema agropecuario y forestal, 
principales actividades productivas del municipio, e incluso muchas han servido de referentes para elaborar diferentes proyectos o implementar ciertos sistemas productivos tanto en la agricultura como la ganadería.

Una investigación relevante fue la realizada en 2002-2003, tesis sobre alternativas de producción de hijos de musáceas de producción acelerada, se logró ganar un proyecto para validar en el campo y hay gente que se apropio de esa tecnología y están produciendo. Fue un trabajo más, pero después de seis meses un organismo le interesó la tecnología y este organismo financió para que se ejecutara el proyecto con los campesinos y la universidad.

Los autores de la monografía tuvieron la oportunidad de brindar asistencia técnica a los productores y replicar esta técnica a una escala más grande en siete comunidades, realización de talleres entre otros, e inclusive se adoptó como una práctica productiva que hasta el día de hoy realizan muchos productores.

Los productores se beneficiaron y se creó una segunda investigación en siete comunidades y esta misma se tomó como modelo y se seleccionó para presentarla en otros eventos.

Al estudiantado, desde el área de investigación y postgrado, se les asigna fondos a través de los cuales realizan investigaciones y de manera puntual se plantean proyectos de estos resultados. Es reciente la preocupación de producir conocimientos sobre el vínculo docencia e investigación, sobre la enseñanza de las ciencias, sobre la pedagogía y las didácticas de las disciplinas, o sobre los estilos pedagógicos, y si bien ya contamos con algunos recursos humanos capacitados en el tema, también es necesario reconocer lo incipiente y la escasez de estímulos para su fortalecimiento.

Aunque existe en el recinto la Dirección de Investigación y Postgrado, y además un inventario de las investigaciones realizadas, a la vez que no se especifica si cumplen o no con las líneas de investigación de la universidad y cuántas de estas entraron con sus últimas sugerencias revisadas. Podría decirse que la investigación, la extensión y la docencia aún no han logrado articularse de manera efectiva, por lo que no se siente a nivel de la sociedad y en la universidad la utilidad de la misma en el mejoramiento de los procesos educativos.

Las entidades locales estatales o privadas, civiles o religiosas, han estado al margen del provecho que pudieran hacer de estas investigaciones, aun cuando existan acuerdos o convenios de la universidad con esas instituciones. Otro aspecto de relevancia encaminado al modelo de investigación propuesto por la URACCAN, es la creación de aspectos normativos de investigación, los cuales hacen referencia a las normas para el desarrollo de la función de investigación, estos se describen en el plan estratégico $2001-2005$. El documento define a la investigación como una función básica y área priorizada de la Universidad. 


\section{Conclusiones}

- Se han generado 115 investigaciones monográficas, siendo las carreras de las áreas de Ciencias de la Educación y Humanidades (71 monografías) y Recursos Naturales y Medio Ambiente (31 monografías), las que han producido más.

- En el 88\% de las investigaciones predomina el enfoque mixto y cuantitativo y únicamente un $12 \%$ se trabajaron bajo el enfoque cualitativo.

- Los tipos de investigación que se destaca son la descriptiva con un 59 \% y la experimental con un $20 \%$.

- La línea de investigación que más se ha trabajado es la de desarrollo socioeconómico ambiental con un 20\%; empero, en las líneas generales no se contemplan otras como por ejemplo las metodologías de enseñanza y aprendizaje que tienen $29 \%$ de los temas investigados.

- A pesar de la variedad de métodos y formas de divulgación, estos no son efectivos, un 59\% del estudiantado conoce de las existencias de las monografías a través del docente; no obstante, se valoran negativamente otras formas de divulgación como murales, internet, papelógrafos, entre otros.

- Siete monografías han sido publicadas en revistas universitarias de URACCAN, lo que equivale a un $6 \%$, dos han sido publicadas a nivel internacional, correspondiendo al $1.73 \%$.

- Las monografías han tenido gran utilidad, como objeto de consultas bibliográficas, generación de proyectos, soluciones de problemáticas relacionadas con el quehacer universitario, municipal y regional, creación de sistemas informáticos, mejoramiento de los procesos de enseñanza aprendizaje, generación de datos y diagnósticos de problemáticas, construcción de nuestra historia municipal, aportes a la preservación del medio ambiente, alternativas de producción y mejoramiento de los sistemas agropecuario y forestal.

- Para el profesorado y estudiantado la información de las monografías sirve como fuentes de datos, generar otras investigaciones y referentes bibliográficos.

- Un $40 \%$ de las monografías existentes en biblioteca son las que tienen mayor demanda, el estudiantado y profesorado obtuvieron un $70 \%$ en consultas de las monografías.

- El estudiantado y profesorado generalmente hacen uso de las monografías, únicamente cuando están cursando asignaturas de metodología de investigación y seminario monográfico o realizan investigaciones para su titulación.

- $\quad$ El 75\% del profesorado entre frecuente y rara vez visitan la biblioteca para revisar las monografías, así mismo un $79 \%$ del profesorado, y el $68 \%$ del estudiantado 
respondieron que los trabajos y tareas asignadas orientan el uso de libros y únicamente un 7\% del profesorado motiva el uso de monografías.

- Para los investigadores de entidades municipales, un $64 \%$ conoce que las monografías han sido útiles y estas son utilizadas para resolver problemáticas relacionadas con la institución u obtener datos que sirven de insumos en otras investigaciones o proyectos que realizan.

- En un $79 \%$ de las instituciones encuestadas, se ha realizado algún tipo de investigación por estudiantado de la universidad, sin embargo sólo en el 50\% de estas se dieron a conocer los resultados de las mismas

- No existe un mecanismo de verificación de la incorporación de las sugerencias que el jurado examinador emite una vez concluida la defensa, para poder ingresar a la biblioteca, se confía esta labor al tutor, la universidad no tiene un mecanismo o se desconoce la forma de cómo estas últimas recomendaciones se incorporan antes de entregarse a biblioteca.

- No existen mecanismos ni normativas donde se especifiquen los indicadores para clasificar estas investigaciones bajo parámetros de calidad, pertinencia, entre otros.

- La universidad ha realizado grandes esfuerzos para fortalecer las capacidades investigativas en docentes y estudiantes a través de seminarios, talleres, postgrados y maestrías.

\section{Lista de referencias}

Asamblea Nacional de la República de Nicaragua (05 de abril de 1990), Ley de autonomía de las instituciones de educación superior. Managua, Nicaragua.

CNU (2004). Evaluación del Desempeño de la Investigación en las Universidades Miembros del CNU. Managua, Nicaragua.

Garza, A. (2006). Los científicos deben escribir. Ciencia y Desarrollo., vol. 32, no. 197.

Henao, M. (2005). El papel de la investigación en la formación universitaria. Recuperado el og de noviembre de 2009, en http://especiales.universia.net.co/docentes/ articulos-de-educacion-superior/el-papel-de-la-investigacion-en-la-formacionuniversitaria.html

UNESCO (1999). Conferencia Mundial sobre la Ciencia y el uso del saber científico. Budapest, Hungría.

UNESCO (1998). Declaración mundial sobre la educación superior en el siglo XXI: visión y acción. Paris, Francia. 